\title{
Un Enfoque Bayesiano en Modelos Heterocedásticos de Series de Tiempo y su Aplicación en la Volatilidad de Activos Financieros
}

\author{
Edwin Antero Flores Montoya $\bigsqcup^{1}$ y Antonio Bravo Quiro $\bigsqcup^{2}$
}

Resumen: En este trabajo, se estudia la modelación de la volatilidad de activos financieros mediante un enfoque bayesiano. Se utilizan modelos DCC - GARCH, para los errores de estos modelos se consideran distribuciones de probabilidad asimétricas y leptocúrticas, las cuales se parametrizan en función de la asimetría y el peso de las colas, por lo que también se estiman estos parámetros. La estimación de los parámetros del modelo se realizó mediante la metodología MCMC algoritmo Metropolis Hastings caminata aleatoria haciendo uso del software $\mathrm{R}$ paquete bayesDccGarch, se consideran datos diarios del 1/04/2015 - 31/01/2020 de los índices bursátiles de: Frankfurt (DAX), Tokio (NIKKEI225), París (CAC40), y de Lima (BVL). El enfoque bayesiano para la estimación de los parámetros del modelo facilita la interpretación y brinda la posibilidad de insertar información a priori para los parámetros.

Palabras clave: Modelos heterocedasticos DCC - GARCH, metodología MCMC.

\section{A Bayesian Approach to Heterocedastic Models of Time Series and its Application in the Volatility of Financial Assets}

\begin{abstract}
In this work, the modeling of the volatility of financial assets is studied using a Bayesian approach. DCC - GARCH models are used, for the errors of these models asymmetric and leptokurtic probability distributions are considered, which are parameterized according to the asymmetry and the weight of the tails, therefore these parameters are also estimated. The estimation of the model parameters was performed using the MCMC methodology Metropolis - Hastings random walk algorithm using the software $\mathrm{R}$ package bayesDccGarch, daily data from 04/01/2015 01/31/2020 of the stock indices of: Frankfurt are considered (DAX), Tokyo (NIKKEI225), Paris (CAC40), and Lima (BVL). The Bayesian approach to estimating the model parameters facilitates interpretation and provides the ability to insert a priori information for the parameters.
\end{abstract}

Keywords: DCC - GARCH heteroscedastic models, MCMC methodology.

Recibido: 11/10/2021. Aceptado: 02/12/2021. Publicado online: 30/12/2021.

\footnotetext{
(C) Los autores. Este artículo es publicado por la Revista PESQUIMAT de la Facultad de Ciencias Matemáticas, Universidad Nacional Mayor de San Marcos. Este es un artículo de acceso abierto, distribuido bajo los términos de la licencia Creative Commons Atribucion-No Comercia-CompartirIgual 4.0 Internacional.(http://creativecommons.org/licenses/by-nc-sa/4.0/) que permite el uso no comercial, distribución y reproducción en cualquier medio, siempre que la obra original sea debidamente citada. Para uso comercial, por favor póngase en contacto con revistapesquimat.matematica@unmsm.edu.pe

${ }^{1}$ UNMSM, Facultad de Ciencias Matemáticas. e-mail: edwinantero.flores@unmsm.edu.pe

${ }^{2}$ UNMSM, Facultad de Ciencias Matemáticas, e-mail:abravoq@unmsm.edu.pe
} 


\section{Introducción}

Los modelos ARCH introducidos por [10], tienen como base fundamental la volatilidad, es decir, la varianza en un instante $t$ no es constante y depende de los retornos pasados.

[7, propuso una clase de modelos que considera que la volatilidad depende, no solo de los retornos pasados, sino que también depende de los valores pasados de la volatilidad, estos son los modelos GARCH, estos modelos son considerados más parcimoniosos pues describen la volatilidad de la serie con menos parámetros.

En la literatura existente hay muchas extensiones multivariadas de los modelos GARCH entre los que se destacan los modelos multivariados CCC - GARCH (correlación condicional constante GARCH) y el modelo DCC - GARCH (correlación condicional dinámica GARCH) propuestos por los mismos creadores de los modelos ARCH y GARCH [8, 11, 21].

Seguidamente surgen algunos enfoques bayesianos para la estimación de los parámetros del modelo GARCH. [3] propone un algorítmo para la estimación bayesiana del modelo $\operatorname{GARCH}(1,1)$ con errores normales, este algorítmo obtiene los valores de las muestras de la distibución a posteriori de los parámetros.

En general, se debe tener en cuenta que en los modelos GARCH las condiciones completas de la a posteriori no coinciden con funciones de densidad de probabilidad conocidas en la literatura debido a la evidencia en la literatura de que muchas series temporales financieras tienden a tener la curtosis observada mayor que aquella considerada en los modelos GARCH con errores normales, 44 proponen la estimación bayesiana del modelo GARCH con errores $t$-Student a través del algoritmo Metropolis - Hastings (MH). Este algoritmo fue propuesto por [17] y luego generalizado por [16], quedando así el algoritmo Metrópolis - Hastings.

[9], compara modelos GARCH con distribuciones asimétricas de los errores. Cabe resaltar que Ehlers utilizó la metodología propuesta por [12] para insertar asimetría mediante los factores de escala inversa en los valores positivos y negativos de la variable, obteniendo así, una distribución asimétrica ajustada solo a un parámetro, de tal manera que éste determina el grado de asimetría para el caso univariado, 5] generalizó esta idea para el caso multivariado.

[15, 13, 14] estudian los modelos GARCH y su principal generalización multivariada, los modelos heterocedásticos DCC - GARCH, para los errores de estos modelos consideraron distribuciones de probabilidad asimétricas y leptocúrticas que se parametrizan de acuerdo con la asimetría y el peso en las colas, por lo que les fué necesario estimar estos parámetros adicionales para sus modelos.

2], consideran dos características principales a ser modeladas: La asimetría y las colas pesadas presentes en la distribución incondicional de las series de retornos, para la estimación de los parámetros se utilizó la metodología MCMC especificamente los algorítmos MH.

[18], recomienda el uso de modelos de bajo orden (por ejemplo: $(1,1),(1,2),(2,1)$ y $(2,2))$ y el modelo se elige según criterios como el: Criterio de información Akaike AIC [1] y el criterio de información Bayesiano BIC [19] entre otros. El autor también afirma que en la mayoría de las series financieras, un modelo $\operatorname{GARCH}(1,1)$ es suficiente para describir bien la volatilidad.

En base a todo lo expuesto y teniendo en cuenta el criterio de Desvio de Información (DIC) propuesto 20]. La motivación del presente trabajo es modelar el indice del mercado accionario empleando la metodología propuesta desde una perspectiva bayesiana. Es decir utilizar la metodología MCMC (algoritmo Metropolis - Hastings caminata aleatoria) haciendo uso del paquete bayesDccGarch implementado en el software R, considerando datos diarios del 1 de abril del 2015 al 31 de enero del 2020 de los índices bursátiles de: Frankfurt (DAX), Tokio (NIKKEI225), París (CAC40), y de Lima (BVL). 


\section{Método Multivariado}

El método para insertar asimetría de [5] generaliza para el caso multivariado el método de [12. La función de densidad es:

$$
s(x \mid \gamma)=2^{m}\left(\prod_{i=1}^{m} \frac{\gamma_{i}}{1+\gamma_{i}^{2}}\right) p\left(x^{*}\right)
$$

donde,

$$
x^{*}=\left(x_{1}^{*} ; x_{2}^{*} ; \ldots ; x_{m}^{*}\right)^{t}=\left\{\begin{array}{c}
\frac{x_{i}}{\gamma_{i}}, \text { si } x_{i} \geq 0 \\
x_{i} \gamma_{i}, \text { si } x_{i}<0
\end{array}\right.
$$

Los parámetros de asimetría $\gamma=\left(\gamma_{1}, \ldots, \gamma_{m}\right)^{t}$, con $\gamma_{i}>0$. Si $\gamma_{i}=1$ la marginal correspondiente es el caso simétrico.

Las medias y las varianzas.

$$
\begin{gathered}
\mu_{i}=E\left(X^{1} \mid \gamma_{i}\right)=\gamma_{i}-\gamma_{i}^{-1} M_{1} \\
\sigma_{i}^{2}=\left(\gamma_{i}^{2}+\gamma_{i}^{-2}-1\right)-\mu_{i}^{2}
\end{gathered}
$$

para $i=1, \ldots, m$. Dada la distribución de probabilidad del vector aleatorio $z=\left(z_{1}, \ldots, z_{m}\right)^{t}$ con $z_{i}=\left(x_{i}-\mu_{i}\right) / \sigma_{i}$, la versión estandarizada de la función de densidad (1) es:

$$
p(z \mid \gamma)=2^{m}\left\{\prod_{i=1}^{m} \frac{\gamma_{i}}{1+\gamma_{i}^{2}} \sigma_{i}\right\} p\left(z^{*}\right)
$$

donde,

$$
z_{i}^{*}=\left\{\begin{array}{l}
\left(z_{i} \sigma_{i}+\mu_{i}\right) \gamma_{i}, \text { si } z_{i}<-\mu_{i} / \sigma_{i} \\
\left(z_{i} \sigma_{i}+\mu_{i}\right) / \gamma_{i}, \text { si } z_{i} \geq-\mu_{i} / \sigma_{i}
\end{array}\right.
$$

En la expresión (4) se obtiene la distribución de probabilidad Normal asimétrica y estandarizada $S S N\left(0, I_{m}, \gamma\right)$,

$$
p(z \mid \gamma)=\left(\frac{2}{\pi}\right)^{\frac{m}{2}}\left(\prod_{i=1}^{m} \frac{\gamma_{i} \sigma_{i}}{1+\gamma_{i}^{2}}\right) \exp \left\{-\frac{1}{2} \sum_{i=1}^{m} z_{i}^{* 2}\right\},
$$

siendo, $z_{i}^{*}$ dado por las expresiones (5).

En la expresión (4) se tiene la distribución de probabilidad $t$-Student asimétrica y estandarizada $S S T\left(0, I_{m}, \gamma, v\right)$,

$$
p(z \mid \gamma, v)=\left(\frac{2}{\sqrt{\pi}}\right)^{m}\left(\prod_{i=1}^{m} \frac{\gamma_{i} \sigma_{i}}{1+\gamma_{i}^{2}}\right) \times \frac{\Gamma\left(\frac{v+m}{2}\right)}{\Gamma\left(\frac{v}{2}\right)(v-2)^{\frac{m}{2}}}\left(1+\frac{\left(z^{*}\right)^{t} z^{*}}{v-2}\right)^{\frac{-(v+m)}{2}}
$$

siendo, $\sigma_{i}$ y $z_{i}^{*}$ dado por las expresiones (3) y (5), respectivamente.

En la expresión (4) se tiene la distribución de probabilidad GED asimétrica y estandarizada $S S G E D\left(0, I_{m}, \gamma, k\right)$.

$$
p(z \mid \gamma, k)=2^{m}\left[\prod_{i=1}^{m} \frac{\gamma_{i} \sigma_{i}}{1+\gamma_{i}^{2}}\right]\left[\frac{\Gamma(3 / k)}{\Gamma(1 / k)}\right]^{m / 2} \times \frac{\exp \left\{-\left[\frac{\Gamma(3 / k)}{\Gamma(1 / k)}\right]^{k / 2} \sum_{i=1}^{m}\left|z_{i}^{*}\right|^{k}\right\}}{(2 / k)^{m}[\Gamma(1 / k)]^{m}}
$$

siendo $\mu_{i}, \sigma_{i}^{2}$ y $z_{i}^{*}$ dados por (2), (3) y (5), respectivamente.

La distribución $S S N$ es en un caso particular de $S S T$, y de $S S G E D$, con $k=2$.

Las distribuciones de probabilidad presentadas en esta sección se aplicarán en los modelos heterocedásticos GARCH multivariados. 


\section{Modelo Heterocedástico Multivariado (DCC - GARCH)}

Sea $y_{t}=\left(y_{t 1}, \ldots, y_{t m}\right)^{t}$ un vector de retornos en el instante $t$ para $m$ series temporales. Así, las extensiones multivariadas de los modelos GARCH se pueden escribir como:

$$
y_{t}=H_{t}^{1 / 2} \epsilon_{t}
$$

siendo $H_{t}$ la matriz de covarianzas condicionales y $H_{t}^{1 / 2}$ la matriz $m \times m$ definida positiva obtenida por la descomposición de Cholesky de la matriz $H_{t}$.

El vector de los errores $\epsilon_{t}$ tiene orden $m \times 1$ y tiene media y varianza dado por:

$$
\begin{aligned}
E\left(\epsilon_{t}\right) & =0 \\
\operatorname{Var}\left(\epsilon_{t}\right) & =I_{m}
\end{aligned}
$$

siendo, $I_{m}$ la matriz identidad de orden $m$. Así, se tiene que la media y la varianza del vector $y_{t}$ condicionado a la información previa hasta el momento $t\left(I_{t}\right)$, son dados por:

$$
\begin{aligned}
E\left[y_{t} \mid I_{t}\right] & =E\left(H_{t}^{1 / 2} \epsilon_{t} \mid I_{t}\right)=H_{t}^{1 / 2} E\left(\epsilon_{t}\right)=0 \\
\operatorname{Var}\left[y_{t} \mid I_{t}\right] & =H_{t}^{1 / 2} \operatorname{Var}\left(\epsilon_{t}\right)\left(H_{t}^{1 / 2}\right)^{t}=H_{t}
\end{aligned}
$$

En la literatura, existen diversas formas de especificar la matriz $H_{t}$ ver [6]. En éste trabajo, se estudian los modelos CC - GARCH (Correlación Condicional GARCH).

[8] propone la primera clase de los modelos $\mathrm{CC}$ - GARCH, los modelos CCC - GARCH (Correlación Condicional Constante GARCH), donde:

$$
H_{t}=D_{t} R D_{t}
$$

con,

$$
D_{t}=\operatorname{diag}\left(h_{11, t}^{1 / 2}, \ldots, h_{m m, t}^{1 / 2}\right)
$$

y $h_{i i, t}$ se define como en un modelo GARCH univariado de cualquier orden. $\operatorname{Si} \operatorname{GARCH}(1,1)$ entonces

$$
h_{i i, t}=\omega_{i}+\alpha_{1, i} y_{i, t-1}^{2}+\beta_{1, i} h_{i i, t-1}
$$

donde $\omega_{i}>0, \alpha_{i} \geq 0, \beta_{i} \geq 0$ y $\left(\alpha_{i}+\beta_{i}\right)<1$ para $i=1, \ldots, m$.

La matriz de correlaciones $R=\left\{\rho_{i j}\right\}_{j=1, \ldots, m}^{i=1, \ldots, m}$ es simétrica y definida positiva con $\rho_{i i}=1$. $\operatorname{Los} \rho_{i j}=\rho_{j i}$, los parámetros adicionales de este modelo serán todos $\rho_{i j}$ con $i>j$, para $i, j=$ $1, \ldots, m$.

De donde se tiene que el número total de parámetros del modelo es $m(m+5) / 2$, el cual crece rápidamente de acuerdo con el número de series. Para 4 series $(m=4)$, se tendrán 18 parámetros, mientras que para 5 series $(m=5), 25$ parámetros.

Seguidamente, fueron propuestos algunos modelos más parcimoniosos entre los que podemos destacar al de [11] y 21] quienes independientemente propusieron el modelo DCC - GARCH (Correlación Condicional Dinámica GARCH) modelo que considera que la matriz de correlación condicional varía en el tiempo.

Adoptando el enfoque de [11] la matriz de covarianzas $H_{t}=D_{t} R_{t} D_{t}$ donde, $D_{t}$ es como en (9) y

$$
R_{t}=\operatorname{diag}\left(Q_{t}\right)^{-1 / 2} Q_{t} \operatorname{diag}\left(Q_{t}\right)^{-1 / 2}
$$

donde $Q_{t}$ son matrices simétricas definidas positivas de orden $m \times m$ dadas por

$$
Q_{t}=(1-a-b) R+a \mu_{t-1} \mu_{t-1}^{t}+b Q_{t-1}
$$

donde $\mu_{t}=D_{t}^{-1} y_{t}, R$ la matriz de covarianzas incondicionales de $\mu_{t}$ y las restricciones de estacionaridad de los parámetros adicionales del modelo son $a>0, b>0$ y $(a+b)<1$. 
Además de modelar la correlación condicional con variación en el tiempo, el modelo DCC GARCH también tiene la ventaja de añadir sólo dos parámetros independientemente del número de series elegidas. Si se considera este modelo el número de parámetros del será $3 m+2$. Luego, para 2 series $(m=2)$ se tendrá 8 parámetros y si se consideran 4 series $(m=4), 14$ parámetros.

La función de densidad de probabilidad de los retornos en los modelos CC - GARCH se pueden obtener en función de la densidad de probabilidad elegida para los errores $\epsilon_{t}$.

Sea $\theta$ el conjunto de todos los parámetros del modelo, la distribución conjunta de los retornos se escribe como el producto de las distribuciones condicionales, es decir:

$$
p\left(y_{1}, \ldots, y_{T} \mid \theta\right)=p\left(y_{1} \mid \theta\right) p\left(y_{2} \mid \theta, y_{1}\right) p\left(y_{3} \mid \theta, y_{1}, y_{2}\right) \ldots p\left(y_{T} \mid \theta, y_{1}, y_{2}, \ldots, y_{T-1}\right)
$$

De la expresión (12), se obtienen las distribuciones condicionales de los retornos en función de la distribución de los errores:

$$
p\left(y_{T} \mid \theta, y_{1}, y_{2}, \ldots, y_{T-1}\right)=p\left(y_{t} \mid I_{t}\right)=\left|H_{t}\right|^{-1 / 2} p_{\epsilon_{t}}\left(H_{t}^{-1 / 2} y_{t}\right)
$$

para $t=1, \ldots, T$.

Así, teniendo las distribuciones condicionales para los $y=\left\{y_{t}, t=1, \ldots, T\right\}$ retornos en función de la distribución de los errores, obtenemos la función de verosimilitud del conjunto de parámetros $\theta$ que son desconocidos

$$
L(\theta)=p\left(\theta \mid y_{1}, \ldots, y_{T}\right)=\left|H_{1}\right|^{-1 / 2} p_{\epsilon_{1}}\left(H_{1}^{-1 / 2} y_{1}\right) \times \cdots \times\left|H_{T}\right|^{-1 / 2} p_{\epsilon_{T}}\left(H_{T}^{-1 / 2} y_{T}\right)
$$

Es decir,

$$
L(\theta)=p\left(\theta \mid y_{1}, \ldots, y_{T}\right)=\prod_{t=1}^{T}\left|H_{t}\right|^{-1 / 2} p_{\epsilon_{t}}\left(H_{t}^{-1 / 2} y_{t}\right)
$$

Para el enfoque Bayesiano se necesita determinar la distribución a priori de los parámetros del modelo.

\section{Estudio de la Distribución a Priori}

La estimación de los parámetros en este trabajo se hizo mediante el enfoque Bayesiano teniendo como base el modelo heterocedástico GARCH, para ello fueron necesarios las a prioris de los parámetros del modelo. En este estudio, para la estimación de los parámetros del modelo GARCH $(1,1)$ se utilizaron las distribuciones a priori propuestas en [3], estas distribuciones son normales truncadas en el espacio paramétrico de cada uno de los parámetros, además se asume independencia a priori entre parámetros, es decir:

$$
\begin{aligned}
\omega & \sim N\left(\mu_{\omega}, \sigma_{\omega}^{2}\right) I_{(\omega>0)}, \\
\alpha_{1} & \sim N\left(\mu_{\alpha}, \sigma_{\alpha}^{2}\right) I_{(0<\alpha<1)}, \\
\beta_{1} & \sim N\left(\mu_{\beta}, \alpha_{\beta}^{2}\right) I_{(0<\beta<1)}
\end{aligned}
$$

donde, $\mu_{\omega}, \mu_{\alpha}, \mu_{\beta}, \sigma_{\alpha}^{2}, \alpha_{\beta}^{2}$ hiperparámetros.

En el caso, de utilizar una distribución asimétrica, como las presentadas en la (sección 2), es necesario estimar el parámetro de asimetría, en este caso [12] proponen usar una distribución a priori Gamma $(a, b)$ para $\gamma^{2}$.

La idea es elegir los valores de $a$ y $b$ de modo que $E(\gamma)=1$.

$$
\begin{aligned}
& 1=E_{\gamma^{2}}(\gamma)=\frac{b^{a}}{\Gamma(a)} \int_{0}^{\infty} \gamma\left(\gamma^{2}\right)^{a-1} e^{-b \gamma^{2}} d \gamma^{2} \\
& 1=\frac{b^{a}}{\Gamma(a)} \int_{0}^{\infty}\left(\gamma^{2}\right)^{(a+0,5)-1} e^{-b \gamma^{2}} d \gamma^{2} \\
& 1=\frac{b^{a}}{\Gamma(a)} \frac{\Gamma(a+0,5)}{b^{a+0,5}}
\end{aligned}
$$


entonces

$$
b=\left[\frac{\Gamma\left(a+\frac{1}{2}\right)}{\Gamma(a)}\right]^{2}
$$

del resultado anterior se elige el valor de $a$ controlando la varianza a priori y la probabilidad a priori de $\gamma \in(0,1)$, fijando $a=1 / 2$ lleva a $V(\gamma)=\pi / 2-1 \approx 0,57$ y $P(0<\gamma<1) \approx 0,58$, la cual es una elección razonable.

Además, esta particular opción es equivalente a especificar:

$$
\gamma \sim N\left(0,0,64^{-1}\right) I_{(\gamma>0)} .
$$

Sin embargo, cuando se utiliza la distibución $\operatorname{SST}(0,1, \gamma, v)$, presentada en (6) o $\operatorname{SSGED}(0,1, \gamma, k)$ presentada el (7) se debe estimar el parámetro de cola $v$ o $k$, respectivamente.

En estos casos, se utilizará

$$
v \sim N\left(\mu_{v}, \sigma_{v}^{2}\right) I_{(v>2)}
$$

$\mathrm{y}$

$$
k \sim N\left(\mu_{k}, \sigma_{k}^{2}\right) I_{(k>0)}
$$

donde, $\mu_{v}, \mu_{k}, \mu_{v} y \sigma_{k}^{2}$ hiperparámetros.

Tenga en cuenta que $N\left(\mu, \sigma^{2}\right) I_{(a<x<b)}$, con $a<b$ representa la función de densidad de probabilidad

$$
\frac{1}{\phi(b)-\phi(a)} \times \frac{1}{\sqrt{2 \pi \sigma^{2}}} \exp \left\{-\frac{(x-\mu)^{2}}{2 \sigma^{2}}\right\}, x \in(a, b)
$$

$\phi($.$) es la función de densidad acumulada de la distribución normal con media \mu$ y varianza $\sigma^{2}$.

La distribución de probabilidad Normal para las a prioris, facilita la inserción de información en una determinada región de interés a través de los parámetros $\mu$ y $\sigma^{2}$ de la distribución normal $N\left(\mu, \sigma^{2}\right)$, aunque en el caso del truncamiento, estos hiperparámetros no representan la media y la varianza, pero aún así controlan la región con la medida de probabilidad más alta.

Para el caso multivariado se extiende el enfoque presentado para los modelos univariados de los parámetros de volatilidad $\left(\omega, \alpha_{1}\right.$ y $\left.\beta_{1}\right)$ y para el parámetro de asimetría $(\gamma)$, presentada en la (sección 2).

Para ello, se asume independencia a priori entre los parámetros de los modelos. De esta forma se obtiene:

$$
\begin{aligned}
\omega_{i} & \sim N\left(\mu_{\omega_{i}}, \sigma_{\omega_{i}}^{2}\right) I_{\left(\omega_{i}>0\right)}, \\
\alpha_{1, i} & \sim N\left(\mu_{\alpha_{i}}, \sigma_{\alpha_{i}}^{2}\right) I_{\left(0<\alpha_{i}<1\right)}, \\
\beta_{1, i} & \sim N\left(\mu_{\beta_{i}}, \alpha_{\beta_{i}}^{2}\right) I_{\left(0<\beta_{i}<1\right)}, \\
\gamma_{i} & \sim N\left(0,0,64^{-1}\right) I_{\gamma_{i}>0},
\end{aligned}
$$

para $i=1, \ldots, m$.

Las a prioris para los parámetros de correlación $a$ y $b$, y las a prioris de la distribución de los errores del modelo $S S T\left(0, I_{m}, \gamma, v\right)$ o $S S G E D\left(0, I_{m}, \gamma, k\right)$ se definen siguiendo el mismo enfoque anterior:

$$
\begin{aligned}
a & \sim N\left(\mu_{a}, \sigma_{a}^{2}\right) I_{(0<a<1)}, \\
b & \sim N\left(\mu_{b}, \sigma_{b}^{2}\right) I_{(0<b<1)}, \\
v & \sim N\left(\mu_{v}, \sigma_{v}^{2}\right) I_{(v>2)}, \text { si } \epsilon_{t} \sim \operatorname{SST}\left(0, I_{m}, \gamma, v\right), \\
k & \sim N\left(\mu_{k}, \sigma_{k}^{2}\right) I_{(k>0)}, \text { si } \epsilon_{t} \sim \operatorname{SSGED}\left(0, I_{m}, \gamma, k\right),
\end{aligned}
$$

Si $m=1$, se obtiene el modelo GARCH univariado. Pues en este caso, se tendría $D_{t}=\left\{h_{11, t}^{1 / 2}\right\}$ y $R_{t}=\{1\}$, con lo cual $H_{t}=\left\{h_{11, t}\right\}$. 


\section{Estimación de los parámetros del modelo heterocedástico}

Para estimar los parámetros del modelo hetocedástico DCC - $\operatorname{GARCH}(1,1)$ se consideraron dos versiones del algoritmo MH. En el primer algoritmo MH se consideró sólo un bloque conteniendo todos los parámetros y en el segundo algoritmo la actualización de la cadena se realiza con un parámetro por bloques.

Para poder simular en la recta, se consideraron transformaciones para todos los parámetros, considerando como espacio paramétrico al conjunto de los números reales. La distribución candidata fué la distribución Normal centrada en el último valor de la cadena, obteniendose algoritmos MH Caminata Alatoria.

Cuadro 1: Transformaciones adoptadas para cada parámetro para que sean mapeados en la recta real.

\begin{tabular}{|l|l|l|}
\hline Transformación $(\phi)$ & Inversa $\left(\phi^{-1}=\theta\right)$ & Distribución (dist. de los errores) \\
\hline$\phi_{1}=\log (v-2)$ & $\nu=\exp \left(\phi_{1}\right)+2$ & $t$-Student asimetrica estandarizada \\
$\phi_{1}=\log (k)$ & $k=\exp \left(\phi_{1}\right)$ & GED asimetrica estandarizada \\
\hline$\phi_{4(i-1)+2}=\log \left(\gamma_{i}\right)$ & $\gamma_{i}=\exp \left(\phi_{4(i-1)+2}\right)$ & \\
$\phi_{4(i-1)+3}=\log \left(\omega_{i}\right)$ & $\omega_{i}=\exp \left(\phi_{4(i-1)+3}\right)$ & Parámetros de series \\
$\phi_{4(i-1)+4}=\log \left(\frac{\alpha_{i}}{1-\alpha_{i}}\right)$ & $\alpha_{i}=\frac{\exp \left(\phi_{4(i-1)+4}\right)}{1+\exp \left(\phi_{4(i-1)+4}\right)}$ & temporales $i, i=1, \cdots, m$ \\
\hline$\phi_{4(i-1)+5}=\log \left(\frac{\beta_{i}}{1-\beta_{i}}\right)$ & $\beta_{i}=\frac{\exp \left(\phi_{4(i-1)+5}\right)}{1+\exp \left(\phi_{4(i-1)+5}\right)}$ & \\
$\phi_{4 m+1}=\log \left(\frac{a}{1-a}\right)$ & $a=\frac{\exp \left(\phi_{4 m+1}\right)}{1+\exp \left(\phi_{4(m+1)}\right)}$ & Para \\
$\phi_{4 m+2}=\log \left(\frac{b}{1-b}\right)$ & $b=\frac{\exp \left(\phi_{4 m+2}\right)}{1+\exp \left(\phi_{4 m+2}\right)}$ & $m \geq 2$ \\
\hline
\end{tabular}

Sea $\phi=\left(\phi_{1}, \ldots, \phi_{n p}\right)^{t}$ el vector de parámetros transformados y $\phi^{-1}$ la transformación inversa a la escala original $\left(\phi^{-1}=\theta\right)$, la función densidad posterior de $\phi$ es dada por:

$$
\pi_{\phi}(\phi \mid y)=\pi\left(\phi^{-1} \mid y\right) J(\phi),
$$

donde $J(\phi)$ es el jacobiano de la transformación:

si $m=1$

$$
J(\phi)=\left|\begin{array}{cccc}
\frac{\partial \theta_{1}}{\partial \phi_{1}} \frac{\partial \theta_{1}}{\partial \phi_{2}} & \cdots & \frac{\partial \theta_{1}}{\partial \phi_{5}} \\
\frac{\partial \theta_{2}}{\partial \phi_{1}} & \frac{\partial \theta_{2}}{\partial \phi_{2}} & \cdots & \frac{\partial \theta_{2}}{\partial \phi_{5}} \\
\vdots & \vdots & \ddots & \vdots \\
\frac{\partial \dot{\theta}_{5}}{\partial \phi_{1}} & \frac{\partial \theta_{5}}{\partial \phi_{2}} & \cdots & \frac{\partial \theta_{5}}{\partial \phi_{n p}}
\end{array}\right|=\frac{e^{\phi_{1}+\ldots+\phi_{5}}}{\left[\left(1+e^{\phi_{4}}\right)\left(1+e^{\phi_{5}}\right)\right]^{2}}
$$

si $m \geq 2$

$$
\left|\begin{array}{cccc}
\frac{\partial \theta_{1}}{\partial \phi_{1}} & \frac{\partial \theta_{1}}{\partial \phi_{2}} & \cdots & \frac{\partial \theta_{1}}{\partial \phi_{n p}} \\
\frac{\partial \theta_{2}}{\partial \phi_{1}} & \frac{\partial \theta_{2}}{\partial \phi_{2}} & \cdots & \frac{\partial \theta_{2}}{\partial \phi_{n p}} \\
\vdots & \vdots & \ddots & \vdots \\
\frac{\partial \theta_{n p}}{\partial \phi_{1}} & \frac{\partial \theta_{n p}}{\partial \phi_{2}} & \cdots & \frac{\partial \theta_{n p}}{\partial \phi_{n p}}
\end{array}\right|=\frac{\prod_{i=1}^{n p} e^{\phi_{i}}}{\left[\left(1+e^{\phi_{4 m+1}}\right)\left(1+e^{\phi_{4 m+2}}\right) \prod_{i=1}^{m}\left(1+e^{\phi_{4(i-1)+4}}\right)\left(1+e^{\phi_{4(i-1)+5}}\right)\right]^{2}}
$$

A continuación se presentan los algoritmos implementados en el software R, paquete bayesDccGarch propuesto por [14]: algoritmo MH 1-bloque y algoritmo MH np-Bloques, donde el espacio paramétrico es $\mathbb{R}^{n p}$, y $n p=4 m+3$ es el número de parámetros del modelo.

\subsection{MH 1-Bloque}

1. Establecer valores iniciales para $\phi_{i}^{(0)}$, donde $i=1, \ldots, n p$ y hacer: $j=0$. 
2. Dejar $\phi^{(j)}=\left(\phi_{1}^{(j)}, \ldots, \phi_{n p}^{(j)}\right)^{t}$, muestra $x \sim N_{n p}\left(0, I_{n p}\right)$ y $u \sim U(0,1)$.

Hacer $\phi^{t}=\phi^{(j)}+\lambda \sum^{1 / 2} x$;

3. Si $u \leq p_{\phi}\left(\phi^{t}, \phi^{(j)}\right)$, entonces hacer $\phi^{(j+1)}=\phi^{t}$, caso contrario $\phi^{(j+1)}=\phi^{(j)}$

4. Hacer $j=j+1$ y regresar a 2 .

Como la distribución generadora de $\phi^{t}$ elegida, es la normal multivariada con media $\phi^{(j)}$ y la densidad es la a posteriori de $\phi$, la probabilidad de aceptación es dado por:

$$
p_{\phi}\left(\phi^{t}, \phi^{(j)}\right)=\min \left\{1, \frac{\pi\left(\phi^{t} \mid y\right)}{\pi\left(\phi^{(j)} \mid y\right)}\right\}
$$

siendo,

$$
\pi(\phi \mid y)=L(\phi) \prod_{i=1}^{n p} p\left(\phi_{i}\right)
$$

y $\sum^{1 / 2}$ es la descomposición de Cholesky de la matriz de varianza - covarianza de $\phi$. El coeficiente $\lambda>0$ es útil para ajustar la tasa de aceptación a valores adecuados (entre $20 \%$ y $50 \%$ para el paquete bayesDccGarch). Como la estimación de $\sum$ se realiza a priori, encontrar una buena aproximación es lo más difícil involucrado en el uso del algoritmo MH 1-Bloque. En el paquete bayesDccGarch hay dos estrategias implementadas para esta propuesta.

El primero usa la siguiente aproximación,

$$
\widehat{\sum}^{-1 / 2}=H^{-1 / 2}
$$

donde $H$ es la matriz Hessiana de la distribución de densidad a posteriori de $\phi$. Sin embargo, pueden surgir problemas computacionales al calcular esta aproximación, en cuyo caso se adopta la segunda estrategia que simula un parámetro por bloque para construir una muestra piloto. A continuación, se calcula una matriz de varianza y covarianza de muestra a partir de la salida de este algoritmo. Este algoritmo se describe a continuación.

\section{2. $\quad$ MH np-Bloques}

1. Establecer valores iniciales para $\phi_{i}^{(0)}$, para $i=1, \ldots, n p$ y hacer: $j=0$.

2. Para $i=1$ hasta $i=n p$ hacer: muestra $x \sim N(0,1)$ y $u \sim U(0,1)$.

Hacer $\phi_{i}^{t}=\phi_{i}^{(j)}+\sigma_{i} x$

Si $u \leq p_{\phi_{i}}\left(\phi_{i}^{t}, \phi_{i}{ }^{(j)}\right)$, entonces hacer $\phi_{i}{ }^{(j+1)}=\phi_{i}^{t}$.

Caso contrario $\phi_{i}^{(j+1)}=\phi_{i}^{(j)}$

3. Hacer $j=j+1$ y regresar a 2 .

Siendo la probabilidad de aceptación $p_{\phi_{i}}\left(\phi_{i}^{t}, \phi_{i}{ }^{(j)}\right)$ dado por

$$
p_{\phi_{i}}\left(\phi_{i}^{t}, \phi_{i}^{(j)}\right)=\min \left\{1, \frac{\pi\left(\phi_{i}^{t} \mid \phi_{i}^{(j)}, y\right)}{\pi\left(\phi_{i}^{(j)} \mid \phi_{i}{ }^{(j)}, y\right)}\right\},
$$

donde

$$
\pi\left(\phi_{i}^{(j)} \mid \phi_{i}^{(j)}, y\right) \propto L\left(\phi_{1}^{(j+1)}, \ldots, \phi_{i-1}^{(j+1)}, \phi_{i}^{(t)}, \phi_{i+1}^{(j)}, \ldots, \phi_{n p}^{(j)}\right) \pi\left(\phi_{i}^{(t)}\right)
$$

El esquema MH np-Bloques también implica la estimación previa de la desviación estándar de los parámetros $\sigma_{1} ; \cdots ; \sigma_{n p}$ para encontrar una tasa de aceptación adecuada para cada parámetro. Aunque es más costoso computacionalmente, esto suele ser más fácil de aproximar que $\sum$. Para más detalles de la implementación del paquete bayesDccGarch ver [14]. 


\section{Aplicación en la volatilidad de activos financieros}

Se consideran los datos de las bolsas de valores de cierre diario de los índices bursátiles de Frankfurt (DAX), Tokio (NIKKEI225), París (CAC40) y de Lima (BVL). Analizados en el periodo del 01 de abril del 2015 al 31 de enero del 2020 totalizando 1263 días observados. Los rendimientos considerados se multiplicaron por 100.

Es decir, si $I_{t}$ es el valor del $i$ - ésimo índice en el tiempo $t$, entonces modelamos la serie multivariante de rendimientos $y_{i t}=100 \log \left(\frac{I_{i t}}{I_{i, t-1}}\right)$.

Para la selección del mejor modelo, se consideran 3 criterios, los dos primeros consisten en la obtención de la esperanza a posteriori de los criterios de información Akaike (EAIC) y Bayesiano (EBIC), el tercer criterio utlizado es la Desviación de Información (DIC). Estos criterios penalizan la función de verosimilitud del modelo de acuerdo con su complejidad, de modo que los mejores modelos tienen un mayor valor de función de verosimilitud y menos parámetros, donde los valores más bajos indican mejores modelos.

Se verificó la convergencia y la buena mezcla de las cadenas de Markov simuladas mediante inspección visual de las trazas marginales, estimaciones de densidad, autocorrelaciones y pruebas formales, estos diagnósticos indicaron buena convergencia, el tiempo de cálculo en este paso fué inferior a 30 minutos para cada modelo además las tasas de aceptación fueron de 0.24 para SST, 0.23 para SSGED y 0.27 para SSN; es decir los tiempos de ejecución son razonables y las tasas de aceptación se encontraron alrededor de 0.234 (propuesta hecha por [22]). El cuadro 2 presenta los criterios de selección del mejor modelo heterocedástico condicionalmente autorregresivo generalizado multivariado correlación condicional dinámica DCC - $\operatorname{GARCH}(1,1)$, para cada una de las distribuciones de probabilidad de los errores estudiados. En este cuadro se puede ver que los criterios EAIC EBIC y DIC, es decir los 3 criterios seleccionaron el modelo con errores SST como mejor modelo.

Cuadro 2: Criterios para la selección del mejor modelo multivariado

\begin{tabular}{|l|l|l|l|}
\hline Modelos & EAIC & EBIC & DIC \\
\hline SSN & 2702.699 & 2795.242 & 2683.934 \\
SST & 2336.367 & 2434.050 & 2316.101 \\
SSGED & 2422.065 & 2519.749 & 2401.647 \\
\hline
\end{tabular}

En el cuadro 3 se muestra un resumen de las simulaciones de MCMC para el modelo con errores SST. Las medias posteriores, desviaciones estandar medianas e intervalos creíbles al $95 \%$ de los parámetros de asimetría indican una alta asimetría para los retornos de las series DAX y NIKKEI225, mientras que para los retornos de CAC40 y para BVL hay una leve inclinación hacia la izquierda.

Además, la estimación del parámetro de cola $\nu$ (última fila del cuadro 3) indica que una distribución de cola gruesa es apropiada para el término de error.

Teniendo en cuenta los resultados del cuadro 3 el modelo heterocedástico de series de tiempo que modela la volatilidad de activos financieros para los datos diarios de las series estadísticas de las bolsas de valores de cierre diario de los índices bursátiles de Frankfurt (DAX), Tokio (NIKKEI225) y París (CAC40) y del índice General de la Bolsa de Valores de Lima (BVL) utilizando el enfoque bayesiano es:

$$
H_{t}=D_{t} R_{t} D_{t} \text { siendo, }
$$

$$
D_{t}=\operatorname{diag}\left(h_{11, t}^{1 / 2}, \ldots, h_{44, t}^{1 / 2}\right)
$$

es decir según los valores de la Tabla 4.2:

$$
h_{11, t}=0,016850+0,073159 y_{1, t-1}^{2}+0,849125 h_{11, t-1}
$$


Cuadro 3: Resumen de la simulaciones MCMC para el modelo heterocedástico con errores SST

\begin{tabular}{|ll|l|c|l|l|l|}
\hline & & Media & Des. Estandar & $2.5 \%$ & Mediana & $97.5 \%$ \\
\hline DAX & $\gamma_{1}$ & 0.939881 & 0.024452 & 0.891326 & 0.940202 & 0.98703 \\
& $\omega_{1}$ & 0.016850 & 0.004701 & 0.009687 & 0.016214 & 0.02781 \\
& $\alpha_{1}$ & 0.073159 & 0.013744 & 0.049254 & 0.072128 & 0.10299 \\
& $\beta_{1}$ & 0.849125 & 0.030489 & 0.780214 & 0.852383 & 0.89898 \\
NIKKEI & $\gamma_{2}$ & 0.900824 & 0.026340 & 0.848303 & 0.901216 & 0.95150 \\
& $\omega_{2}$ & 0.022563 & 0.006338 & 0.012567 & 0.021765 & 0.03704 \\
& $\alpha_{2}$ & 0.183121 & 0.036616 & 0.122100 & 0.179810 & 0.26381 \\
& $\beta_{2}$ & 0.731489 & 0.051036 & 0.618716 & 0.736182 & 0.81644 \\
CAC40 & $\gamma_{3}$ & 0.966817 & 0.027869 & 0.912794 & 0.966745 & 1.02204 \\
& $\omega_{3}$ & 0.016825 & 0.004150 & 0.010343 & 0.016296 & 0.02634 \\
& $\alpha_{3}$ & 0.095057 & 0.017027 & 0.065107 & 0.093741 & 0.13242 \\
& $\beta_{3}$ & 0.818483 & 0.032895 & 0.745736 & 0.821863 & 0.87326 \\
BVL & $\gamma_{4}$ & 0.961111 & 0.026679 & 0.908582 & 0.960875 & 1.01454 \\
& $\omega_{4}$ & 0.009713 & 0.003943 & 0.004069 & 0.009053 & 0.01896 \\
& $\alpha_{4}$ & 0.079194 & 0.024085 & 0.039816 & 0.076742 & 0.13401 \\
& $\beta_{4}$ & 0.846237 & 0.048256 & 0.737352 & 0.852563 & 0.92177 \\
\hline & $\mathrm{a}$ & 0.030546 & 0.009782 & 0.013730 & 0.029797 & 0.05182 \\
& $\mathrm{~b}$ & 0.814858 & 0.079481 & 0.624053 & 0.828090 & 0.93177 \\
& $\nu$ & 5.566379 & 0.350145 & 4.897407 & 5.560763 & 6.26530 \\
\hline
\end{tabular}

$$
\begin{gathered}
h_{22, t}=0,022563+0,183121 y_{2, t-1}^{2}+0,731489 h_{22, t-1} \\
h_{33, t}=0,016825+0,095057 y_{3, t-1}^{2}+0,818483 h_{33, t-1} \\
h_{44, t}=0,009713+0,079194 y_{4, t-1}^{2}+0,846237 h_{44, t-1} \\
R_{t}=\operatorname{diag}\left(Q_{t}\right)^{-1 / 2} Q_{t} \operatorname{diag}\left(Q_{t}\right)^{-1 / 2}
\end{gathered}
$$

donde $Q_{t}$ son matrices simétricas definidas positivas de orden $4 \times 4$ dadas por

$$
Q_{t}=(1-0,030546-0,814858) R+0,030546 \mu_{t-1} \mu_{t-1}^{t}+0,814858 Q_{t-1}
$$

Con $\mu_{t}=D_{t}^{-1} y_{t}$ y $R$ la matriz de covarianzas incondicionales de $\mu_{t}$.

Finalmente, es de interés práctico comprobar si las correlaciones cambian con el tiempo, lo que justifica la complejidad adicional que introduce el modelo DCC, esto se puede ver de las estimaciones en la parte inferior del cuadro 3 las cuales indican que la hipótesis del modelo de correlación condicional constante (CCC) es decir $a=b=0$ son rechazadas en base a las distribuciones marginales posteriores de $a$ y $b$, además tieniendo en cuenta la distribución posterior para la suma $a+b$ se ve que hay una persistencia fuerte en la ecuación (11) pues $(a+b=0,030546+0,814858=0,845404)$.

\section{Conclusión}

1. En este trabajo se logró mediante el enfoque bayesiano, metodologiá MCMC, modelar la volatidad de activos financieros. La metodología bayesiana para la estimación de los parámetros de los modelos trajo algunas ventajas, como la facilidad de interpretación y la posibilidad de insertar información a priori para los parámetros. 
2. Con los datos obtenidos de la web, un total de 1263 para cada serie tomados en el periodo del 01 de abril del 2015 al 31 de enero del 2020, se obtuvo los retornos para cada serie y a éstos se les multiplicó por 100, para luego hacer la simulación de las cadenas de Markov mediante el algoritmo Metropolis - Hasting caminata aleatoria en bloques y en un solo bloque, empleando tiempos razonables (menos de 30 minutos). Para lo cual se utilizó las siguientes órdenes en el programa $\mathrm{R}$ paquete bayesDccGarch:

a) Para la distribución de errores SSN:

$\mathrm{SSN}=$ bayesDccGarch $\left(\right.$ datosx100, $\mathrm{nSim}=200000, \nu_{i}=\mathrm{NULL}$,

$\omega_{i}=\operatorname{rep}\left(0.03, \operatorname{ncol}(\right.$ datosx100) $), \alpha_{i}=\operatorname{rep}(0.03, \operatorname{ncol}($ datosx100) $)$,

$\beta_{i}=\operatorname{rep}(0.8, \operatorname{ncol}(\operatorname{datosx} 100)), a_{i}=0.03, b_{i}=0.8, \gamma_{i}=\operatorname{rep}(1, \operatorname{ncol}(\operatorname{datosx} 100))$,

errorDist $=1$, control $=$ list $($ nPilotSim $=10000)$ )

$\mathrm{SSN} 1=$ window $(\mathrm{SSN}$, start $=30000$, end $=200000$, thin $=5)$

summary(SSN1)

b) Para la distribución de errores SST:

$\mathrm{SST}=$ bayesDccGarch $\left(\right.$ datosx100, $\mathrm{nSim}=200000, \nu_{i}=8$, errorDist $=2$

$\mathrm{SST} 1=$ window $(\mathrm{SST}$, start $=30000$, end $=200000$, thin $=5)$ summary(SST1)

c) Para la distribución de errores SSGED:

$\mathrm{SSGED}=$ bayesDccGarch (datosx100, $\mathrm{nSim}=200000, k_{i}=1.5$, errorDist $=3$

SSGED1 $=$ window $($ SSGED, start $=30000$, end $=200000$, thin $=5)$

summary(SSGED1).

Tomando las esperanzas a posterioris de los criterios de información (AIC, BIC y DIC), y según los datos del cuadro 3, el modelo heterocedástico condicionalmente autorregresivo generalizado multivariado correlación condicional dinámica DCC - GARCH $(1,1)$ seleccionado fué el que usó para los errores la distribución de probabilidad SST.

3. El enfoque bayesiano junto con el uso de distribuciones de probabilidad asimétricas brindó la posibilidad de analizar la distribución de probabilidad de asimetría presente en los rendimientos de las series de activos financieros.

\section{Referencias bibliográficas}

[1] Akaike, H. (1974). A new look at the statistical model identification. IEEE transactions on automatic control, 19(6), 716-723.

[2] Aquino Gutierrez, K. F. (2017). Modelagem da volatilidade em séries temporais financeiras via modelos GARCH com abordagem bayesiana.

[3] David, A. (2006). Bayesian Estimation of the GARCH(1, 1) Model with Normal Innovations.

[4] Ardia, D. (2008). Financial risk management with Bayesian estimation of GARCH models. Heidelberg: Springer.

[5] Bauwens, L., \& Laurent, S. (2005). A new class of multivariate skew densities, with application to generalized autoregressive conditional heteroscedasticity models. Journal of Business \& Economic Statistics, 23(3), 346-354.

[6] Bauwens, L., Laurent, S., \& Rombouts, J. V. (2006). Multivariate GARCH models: a survey. Journal of applied econometrics, 21(1), 79-109.

[7] Bollerslev, T. (1986). Generalized autoregressive conditional heteroskedasticity. Journal of econometrics, 31(3), 307-327. 
[8] Bollerslev, T. (1990). Modelling the coherence in short-run nominal exchange rates: a multivariate generalized ARCH model. The review of economics and statistics, 498-505.

[9] Ehlers, R. S. (2012). Computational tools for comparing asymmetric GARCH models via Bayes factors. Mathematics and Computers in Simulation, 82(5), 858-867.

[10] Engle, R. F. (1982). Autoregressive conditional heteroscedasticity with estimates of the variance of United Kingdom inflation. Econometrica: Journal of the Econometric Society, 987-1007.

[11] Engle, R. (2002). Dynamic conditional correlation: A simple class of multivariate generalized autoregressive conditional heteroskedasticity models. Journal of Business \& Economic Statistics, 20(3), 339-350.

[12] Fernández, C., \& Steel, M. F. (1998). On Bayesian modeling of fat tails and skewness. Journal of the American Statistical Association, 93(441), 359-371.

[13] Fioruci, J. A., Ehlers, R. S., \& Andrade Filho, M. G. (2014). Bayesian multivariate GARCH models with dynamic correlations and asymmetric error distributions. Journal of Applied Statistics, 41(2), 320-331.

[14] Fioruci, J. A., Ehlers, R. S., \& Louzada, F. (2014). BayesDccGarch-An Implementation of Multivariate GARCH DCC Models. arXiv preprint arXiv:1412.2967.

[15] Fioruci, J. A. (2012). Modelagem de volatilidade via modelos GARCH com erros assimétricos: abordagem Bayesiana (Doctoral dissertation, Universidade de São Paulo).

[16] Hastings, W. K. (1970). Monte Carlo sampling methods using Markov chains and their applications.

[17] Metropolis, N., Rosenbluth, A. W., Rosenbluth, M. N., Teller, A. H., \& Teller, E. (1953). Equation of state calculations by fast computing machines. The journal of chemical physics, 21(6), 1087-1092.

[18] Morettin, P. A. (2017). Econometria financeira: um curso em séries temporais financeiras. Editora Blucher.

[19] Schwarz, G. (1978). Estimating the dimension of a model. The annals of statistics, 6(2), 461-464.

[20] Spiegelhalter, D. J., Best, N. G., Carlin, B. P., \& Van Der Linde, A. (2002). Bayesian measures of model complexity and fit. Journal of the royal statistical society: Series b (statistical methodology), 64(4), 583-639.

[21] Tse, Y. K., \& Tsui, A. K. C. (2002). A multivariate generalized autoregressive conditional heteroscedasticity model with time-varying correlations. Journal of Business \& Economic Statistics, 20(3), 351-362.

[22] Roberts, G. O., \& Rosenthal, J. S. (2001). Optimal scaling for various Metropolis-Hastings algorithms. Statistical science, 16(4), 351-367. 